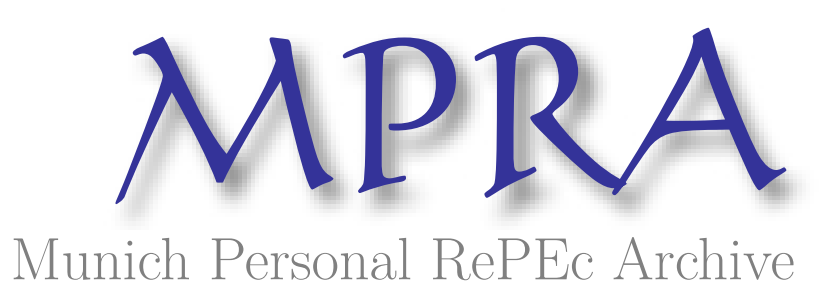

\title{
On the Distribution of Money Holdings in a Random-Matching Model
}

Berentsen, Aleksander

University of Basel

2002

Online at https://mpra.ub.uni-muenchen.de/37319/

MPRA Paper No. 37319, posted 13 Mar 2012 15:03 UTC 


\title{
On the Distribution of Money Holdings in a Random-Matching Model*
}

\author{
Aleksander Berentsen ${ }^{1}$ \\ Economics Department, University of Basel, Switzerland \\ Shortened Title: On the Distribution of Money Holdings
}

\begin{abstract}
This paper studies stationary and nonstationary distributions of money holdings in a random-matching model. The first part characterizes the stationary distributions of money holdings and derives the optimum quantity of money. The second part considers nonstationary distributions of the optimum quantity of money to show that if the production costs are not too large, any distribution of the optimum quantity of money converges asymptotically to the uniform distribution.
\end{abstract}

\section{Keywords}

Optimum Quantity of Money, Distribution of Money Holdings, Convergence, Search Equilibrium

\section{JEL}

D51, E40, E52

\footnotetext{
${ }^{0}$ Submission: February 28, 2000; Revision: November 28, 2000; Accepted: December 14, 2000

${ }^{1}$ I wish to thank Ernst Baltensperger, Marianne Bürgi, Miguel Molico, Guillaume Rocheteau, Randall Wright, Ruilin Zhou, and two anonymous referees for their very valuable comments. I also gratefully acknowledge financial support from a grant received by the Swiss National Science Foundation.
} 


\section{Introduction}

This paper extends the basic search-theoretic model of money developed by Kiyotaki and Wright $(1991,1993)$ by allowing agents to accumulate money up to the bound $n \in \mathbb{N}^{+}$. First, it generalizes the result obtained in Kiyotaki and Wright (1991, 1993) that the optimal quantity of money is $1 / 2$ when the upper bound is $n=1$ to show that the optimal quantity of money is $n / 2$ when the upper bound is $n$. Second, it demonstrates that if the production cost are not too large, any initial distribution of the optimum amount of money converges asymptotically to the uniform distribution.

Following the seminal work of Kiyotaki and Wright (1991, 1993), several articles have either relaxed the fixed price assumption (Trejos and Wright, 1995; Shi, 1995; Berentsen, Molico, and Wright, 2000), the one-unit constraint on money holdings (Berentsen, 2000; Rocheteau, 2000), or both (Corbae and Camera, 1999; Green and Zhou, 1998a; Molico, 1998; Taber and Wallace, 1999; Zhou, 1999). A further line of research has developed tractable versions of the search framework with fully divisible money (Berentsen and Rocheteau, 2000; Shi, 1997, 1999; Lagos and Wright, 2001).

This paper relaxes the one-unit constraint on money holdings, but money and commodities must still exchange one for one. This intermediate step is interesting in its own

right. First, it allows for an analysis of nonstationary distributions of money holdings that - with the exception of Green and Zhou (1998b) — has not been carried out yet. Second, it complements the analysis of stationary equilibria by Corbae and Camera (1999) and Zhou (1999), where commodities are exchanged for one indivisible unit of money, by focusing on the welfare properties of these equilibria. 


\section{The model}

The economy is populated by a $[0,1]$ continuum of infinite-lived agents who specialize in consumption and production. The commodities are indivisible and are nonstorable (to rule out commodity money). Let $X_{i}$ be the set of goods that agent $i$ consumes. No agent $i$ produces a good in $X_{i}$. Moreover, for a pair of agents $i$ and $j$ selected at random, the probability that $i$ produces a good in $X_{j}$ and also $j$ produces a good in $X_{i}$ is 0 (there are no double coincidences of real wants), while the probability that $i$ produces a good in $X_{j}$ but $j$ does not produce a good in $X_{i}$ is $x \in(0,1)$. For example, if there are $J$ goods and $J$ types, $J>2$, and each type $i$ agent consumes only good $i$ and produces only good $i+1$ $(\bmod J)$, then $x=J^{-1}$.

Consuming one unit of a consumption good in $X_{i}$ yields utility $U>0$. Consuming one of the other commodities yields zero utility. Production of one unit of a real commodity $\operatorname{costs} C$ with $U>C \geq 0$. In addition to the consumption goods, there is also an object called fiat money. Fiat money comes in indivisible units of size one, is storable, and cannot be consumed by any agent. Agents can accumulate money up to the bound $n \in \mathbb{N}^{+}$.

The model is in continuous time, and agents meet according to a Poisson process with arrival rate $\alpha$. Total population is normalized to one, and the measure of agents of each type is the same, which implies that the rate at which an agent meets other agents of a particular type is $\alpha x$. Denote by $m_{i}(t)$ the probability that at time $t$ a randomly chosen agent has accumulated $i$ units of money, and denote by $m(t)=\left\{m_{0}(t), \ldots, m_{n}(t)\right\}$ a probability

measure (satisfying $\sum_{i=0}^{n} m_{i}(t)=1$ ). The probability that a randomly selected agent has accumulated less than $n$ units of money is $1-m_{n}(t)$, and the probability that he has accumulated at least one unit of money is $1-m_{0}(t)$. Accordingly, the rate at which an agent meets a potential buyer is $p_{b}=\alpha x\left(1-m_{n}(t)\right)$, and the rate at which he meets a potential seller is $p_{s}=\alpha x\left(1-m_{0}(t)\right)$. 
Throughout the paper the quantity of money, $M$, is assumed to be constant. Note that for any $n$ the quantity of money is bounded in $[0, n]$ : If no agent holds money, the quantity of money is zero, and if all agents hold $n$ units of money, the quantity is $n$. Moreover, at any point of time the probability measure $m(t)$ must satisfy

$$
M=\sum_{i=0}^{n} i m_{i}(t) .
$$

As in Kiyotaki and Wright $(1991,1993)$, money is indivisible and agents cannot hold more than one unit of money when they search for a trading opportunity. ${ }^{2}$ However, in contrast to their models, agents are able to accumulate money by storing their money holdings at home. This assumption makes the pricing decision very simple, because two matched agents either exchange money for goods one for one or do not trade at all. This simplification allows us to focus on the welfare and convergence properties of the model.

In this section, stationary equilibria where $m_{i}(t)=m_{i}$ are considered only. Denote the expected utility (value function) of an agent with money holdings $i, i=0, \ldots, n$, by $V^{i}$. Then, if $r$ is the rate of time preferences, the value functions satisfy

$$
\begin{gathered}
r V^{0}=p_{s} \max \left\{V^{1}-V^{0}-C, 0\right\} \\
r V^{i}=p_{b} \max \left\{U+V^{i-1}-V^{i}, 0\right\}+p_{s} \max \left\{V^{i+1}-V^{i}-C, 0\right\}, i=0, \ldots, n-1, \\
r V^{n}=p_{b} \max \left\{U+V^{n-1}-V^{n}, 0\right\}
\end{gathered}
$$

For example, the first equation sets the flow value of being an agent with no money, $r V^{0}$, equal to the rate at which he meets an agent who buys his product, $p_{s}$, times the gain of either producing for money or refusing to do so.

Definition 1 For any $n>0$ and $M \in(0, n)$, a stationary monetary equilibrium is a list $\langle V, m\rangle$ that satisfies the following conditions:

\footnotetext{
${ }^{2}$ Berentsen and Rocheteau (2001) analyse the implications of indivisible money for the efficiency of monetary exchange in the Kiyotaki-Wright framework.
} 
i) $V$ satisfies (1) taking the probability measure $m$ as given,

ii) the probability measure $m$ is stationary taking the values function $V$ as given, and iii) $V>0$.

According to the first part of Definition 1, the monetary equilibrium is a Nash equilibrium for a given probability measure $m$. The second part requires that the economy be in a steady state given the selling and buying activities induced by equations (1). The third part requires that money have value.

Lemma 1 establishes the existence and uniqueness of a stationary distribution of money (stationary probability measure $m$ ) when agents accumulate money up to the bound $n$.

Lemma 1 For any $n$ and $M \in(0, n)$, if agents accumulate money up to the bound $n$, there is a unique stationary probability measure $m$ which satisfies

$$
\begin{gathered}
m_{i}=m_{0}^{\left(\frac{n-i}{n}\right)} m_{n}^{\left(\frac{i}{n}\right)}, i=0, \ldots, n \\
\sum_{i=0}^{n} m_{0}^{\left(\frac{n-i}{n}\right)} m_{n}^{\left(\frac{i}{n}\right)}=1 \\
\frac{m_{n}}{m_{n-1}}=\frac{m_{n-1}}{m_{n-2}}=\ldots=\frac{m_{1}}{m_{0}}=\frac{1-m_{0}}{1-m_{n}}
\end{gathered}
$$

Proof: See appendix.

Two comments are in order here. First, a similar characterization of the stationary distribution of money holdings has been independently developed by Zhou (1999) and Camera and Corbae (1999). Second, the uniqueness result is derived by the assumption that agents cannot spend more than one unit of money at a time. Without this restriction, there may be many other stationary distributions, including a similar class of equilibria where agents treat $j<n$ units of money as one (Zhou 1999).

It is well established that in this model when $C>0$ there is an endogenous upper bound $I$ (Berentsen 2000). The existence of $I$ is due to two properties of the model. First, 
the marginal expected utility of money is a monotonically decreasing function of money holdings. Second, the cost of acquiring one unit of money (the production $\operatorname{cost} C$ ) is constant. For small money holdings, the increase in the expected utility outweighs the cost, and for large money holdings, the cost is larger than the benefit. Accordingly, if $C$ is large, $I<n$ and agents are only willing to sell for money when $i<I$.

Lemma 2 For any $n$ and money supply $M \in(0, n)$, if $C \leq \tilde{C}$, where $\tilde{C}>0$ is defined in the proof, a unique stationary monetary equilibrium exists.

Proof: See appendix.

According to Lemma 2, for any exogenous upper bound $n$ and any quantity of money $M$, if the production costs are not too large, agents are willing to accumulate money up to the bound $n$. The uniqueness of the equilibrium is a consequence of Lemma 1 .

In the following, for a given bound $n$ the optimum amount of money and its unique stationary distribution are derived. Welfare is defined by $W(M, n)=\sum_{i=0}^{n} m_{i} V^{i}$, which measures the ex ante expected utility of all agents (or a single agent) before money is distributed among them.

Proposition 1 For any $n$ and money supply $M \in(0, n)$, in a stationary monetary equilibrium $r W(M, n)=\alpha x\left(1-m_{n}\right)\left(1-m_{0}\right)(U-C)$. Moreover, the quantity of money $M=\frac{n}{2}$ and the distribution $m_{i}=\frac{1}{n+1}, i=0, \ldots, n$, maximize $W(M, n)$.

Proof: Multiply each value function of (1) by its measure and then add the value functions to get

$$
r W(M, n)=\alpha x\left(1-m_{n}\right)\left(1-m_{0}\right)(U-C)-\sum_{i=1}^{n}\left(p_{b} m_{i}-p_{s} m_{i-1}\right)\left(V^{i}-V^{i-1}\right)
$$

In a stationary monetary equilibrium $\left(p_{b} m_{i}-p_{s} m_{i-1}\right)=0, i=1, \ldots, n$. Hence, $r W(M, n)=$ $\alpha x\left(1-m_{n}\right)\left(1-m_{0}\right)(U-C)$. Next, maximize $W(M, n)$ with respect to $m_{0}$ subject to $(3)$ 
to get $m_{0}=m_{n}$. Given this, Lemma 1 immediately implies that $m_{i}=\frac{1}{n+1}, i=0, \ldots, n$. Accordingly, the optimum quantity of money is $\frac{n}{2}$.

The intuition for this result is straightforward. No trade takes place between an appropriate seller and an appropriate buyer if and only if either the buyer has no money or the seller has money holdings $n$. Consequently, welfare is maximized when the measure of agents in either of these two states is minimized, and this is attained with the uniform distribution $m_{i}=\frac{1}{n+1}, i=0, \ldots, n$. The uniform distribution then immediately implies that the optimum quantity of money is $n / 2$. Note that Proposition 1 generalizes Kiyotaki and Wright's $(1991,1993)$ welfare analysis. In their models, when no barter trade is possible, the optimum amount of money is $1 / 2 .^{3}$

In Proposition 2 welfare maximization is constrained to stationary distributions of money. There are nonstationary distributions of the optimum quantity of money that can temporarily increase (but also decrease) the frequency of trades (and accordingly welfare). This increase, however, is temporal, because the distribution of money converges to the unique stationary distribution associated with the optimum quantity of money, as shown in Section 3.

\footnotetext{
${ }^{3}$ Note that the optimum quantities of money and welfare are strictly increasing in $n$. Thus, a social planner would choose $n=I$. Moreover, when $r \rightarrow 0, I \rightarrow \infty$, which implies that when $r \rightarrow 0$ the optimum quantity of money becomes infinitely large. In this limiting economy, almost no agent is constrained by his money holdings. This result relates to Friedman's (1969) observation that an efficient monetary system requires that agents be constrained by their average flow of income, but not by immediate shortages of cash.
} 


\section{Convergence}

This section studies the convergence property of the model when the optimum quantity of money is distributed. The convergence property is of interest because it gives us some intuition of whether the distribution of money matters in the long run. During the transition the endogenous bound $I(t)$ is time dependent. If for all $t$ we have $I(t) \geq n$, the law of motion proceeds according to the following system of nonlinear differential equations:

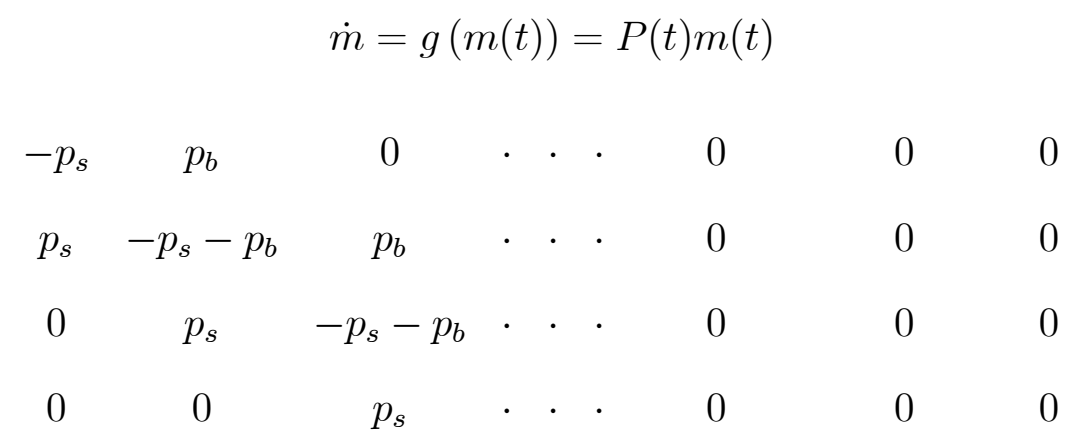

where $P(t)=$

\begin{tabular}{|c|c|c|c|c|c|}
\hline 0 & 0 & 0 & . . . & $p_{b}$ & 0 \\
\hline 0 & 0 & 0 & . . . & $\cdot-p_{s}-p_{b}$ & $p_{b}$ \\
\hline 0 & 0 & 0 & . . . & $p_{s}$ & $-p_{s}-p_{b}$ \\
\hline 0 & 0 & 0 & . . . & 0 & $p_{s}$ \\
\hline
\end{tabular}

Proposition 2 For any $n$, there is a critical value $\bar{C}>0$ defined in the proof such that if $C \leq \bar{C}$, any initial distribution of the optimum amount of money $\frac{n}{2}$ converges asymptotically to the uniform distribution.

Proposition 2 provides a sufficient condition that guarantees convergence to the stationary distribution from an arbitrary distribution of the optimum quantity of money. The sufficient condition is essentially a restriction on the production cost that guarantees that the exogenous bound on money holdings, $n$, remains binding at all times, i.e., that 
$I(t)>n$ for all $t$. The first part of the proof of Proposition 2 involves Liapounov's second method, which is described in Lemma $3 .{ }^{4}$ Denote the gradient vector, $\boldsymbol{\nabla} f(m(t))$, by $\boldsymbol{\nabla} f(m(t))=\left(\frac{\partial f}{\partial m_{0}}, \ldots, \frac{\partial f}{\partial m_{n}}\right)$.

Lemma 3 Let $X \subset \Re^{s}$ be compact, and let $g: X \rightarrow X$ be continuous with $g(\bar{m})=\bar{m}$ for some $\bar{m} \in X$. Suppose there exists a continuous function $f: X \rightarrow \Re$ that satisfies

a) $f(m(t)) \geq 0$, with equality if and only if $m(t)=\bar{m}$,

b) $\dot{f}(m(t))=\boldsymbol{\nabla} f(m(t)) g(m(t)) \leq 0$, with equality if and only if $m(t)=\bar{m}$.

Then $\bar{m}$ is a globally stable solution to the set of nonlinear differential equations $\dot{m}=$ $g(m(t))$.

Proof of Proposition 2: The proof involves two steps. First, it is shown that (5) is globally (asymptotically) stable if on the equilibrium path at any point of time $I(t) \geq n$. Second, a sufficient condition is derived that guarantees that during the entire transition to the stationary distribution one has $I(t) \geq n$.

First step. To prove the global stability of (5) when $I(t) \geq n$, one has to find a Liapounov function that is a continuous function and that satisfies conditions a) and b) of Lemma 3. In the following I show that the function $f(m)=\sum_{i=0}^{n}\left(m_{i}(t)-\bar{m}_{i}\right)^{2}$ satisfies these conditions. Note, first, that $X=\left\{m \in I^{n+1}: \sum_{i=0}^{n} m_{i}=1\right.$ and $\left.\sum_{i=0}^{n} i m_{i}=\frac{n}{2}\right\}$ and that $X$ is compact and convex. $f(m)$ is continuous, and condition a) is satisfied. Condition b) implies

$$
\dot{f}=\sum_{i=0}^{n} 2\left(m_{i}-\bar{m}_{i}\right) \dot{m}_{i} \leq 0
$$

Because $\sum_{i=0}^{n} \bar{m}_{i} \dot{m}_{i}=\bar{m} \sum_{i=0}^{n} \dot{m}_{i}=0,(6)$ is reduced to $\sum_{i=0}^{n} m_{i} \dot{m}_{i} \leq 0$. Use (5) to get

$$
p_{s}\left(\sum_{i=0}^{n-1} m_{i}^{2}-\sum_{i=0}^{n-1} m_{i} m_{i+1}\right)+p_{b}\left(\sum_{i=1}^{n} m_{i}^{2}-\sum_{i=0}^{n-1} m_{i} m_{i+1}\right) \geq 0
$$

\footnotetext{
${ }^{4}$ The proof of Lemma 3 can be found, for example, in Stokey and Lucas (1989, p. 139ff.).
} 
After manipulations of (7) one gets

$$
\left(2-m_{0}-m_{n}\right) \sum_{i=0}^{n-1}\left(m_{i}-m_{i+1}\right)^{2} \geq\left(m_{0}-m_{n}\right)^{2}\left(m_{0}+m_{n}\right)
$$

Denote by $L H S(m)(R H S(m))$ the left-hand (right-hand) side of (8). For any $m_{0}$ and $m_{n}$, $L H S(m)$ is minimized when $m_{i}=\frac{(n-i) m_{0}+i m_{n}}{n}, i=1, . ., n-1$. To see this, differentiate $\sum_{i=0}^{n-1}\left(m_{i}-m_{i+1}\right)^{2}$ with respect to $m_{i}, i=1, . ., n-1$, to get $m_{i}=\frac{m_{i-1}+m_{i+1}}{2}$. Solve these equations to get

$$
m_{i}=\frac{(n-i) m_{0}+i m_{n}}{n}, i=0, . ., n .
$$

Denote by $\tilde{X}$ the set of all probability measures that solve (9). Next, note that for any $m \in \tilde{X},(8)$ holds with equality. This implies that for any $m \in X, m \notin \tilde{X}, m_{0}=\tilde{m}_{0}$, and $m_{n}=\tilde{m}_{n}$

$$
L H S(m)>L H S(\tilde{m})=R H S(\tilde{m})=R H S(m)
$$

To proceed, use $\sum_{i=0}^{n} m_{i}=1$ and (9) to get

$$
\left(m_{0}+m_{n}\right)(n+1)=2
$$

Next, $\sum_{i=0}^{n} i m_{i}=\frac{n}{2}$ and $(9)$ yield

$$
\frac{(1+n)\left(m_{n}(1+2 n)+m_{0}(n-1)\right)}{6}=\frac{n}{2}
$$

The unique solution to (11) and (12) is $m_{0}=m_{n}=\frac{1}{n+1}=\bar{m}$, and accordingly we have $m_{i}=\bar{m}, i=1, . ., n$, and (8) holds with equality, as required by condition b). This result and (10) confirm that $f(m)$ satisfies condition b). Hence, $f(m)$ is a Liapounov function.

Second step. During the transition the endogenous bound $I(t)$ could fall below $n$. In the following a bound on $C$ is derived, denoted by $\bar{C}$, such that if $C \leq \bar{C}$, at any point of time we have $I(t) \geq n$. For any initial distribution $m$ of the optimum quantity of money $\frac{n}{2}$, denote by $C_{m}$ the value of $C$ such that $h(m)=V^{n}(m)-V^{n-1}(m)=C$. If $C \leq C_{m}$, then $h(m) \geq C$. To see that $h(m)>0$ note that at any point of time we have 
$p_{s}=\alpha x\left(1-m_{0}\right)>0$ because if $m_{0}=1$ then $M<\frac{n}{2}$, and $p_{b}=\alpha x\left(1-m_{n}\right)>0$ because if $m_{n}=1$ then $M>\frac{n}{2}$. Denote by $\hat{m}$ the initial distribution of the optimum quantity of money that minimizes $h(m)$, and denote by $\bar{C}$ the value of $C$ such that $h(\hat{m})=C$. Because $p_{s}(\hat{m}), p_{b}(\hat{m})>0$, we have $\bar{C}>0$. If $C \leq \bar{C}$, then $I(\hat{m}) \geq n$. Because $\hat{m}$ minimizes $h(m)$, for all $m$ we have $h(m) \geq \bar{C}$, and accordingly, if $C \leq \bar{C}$, then $I(m) \geq n$ for all $m$. Thus, if $C \leq \bar{C}$, at any point of time we have $I(t) \geq n$. Consequently, any initial distribution of the optimum amount of money converges asymptotically to the uniform distribution.

Three comments are in order here. First, the condition $C \leq \bar{C}$ is sufficient but not necessary for convergence. There are distributions that converge to the uniform distribution even when $C>\bar{C}$. Second, as for the characterization of the stationary equilibria, the uniqueness result is derived by assuming that during the transition goods and money must exchange one for one. Without this assumption multiple equilibria are likely to occur. Third, a redistribution of money affects welfare, although only temporarily. The welfare effect is ambiguous and depends on the initial distribution. 


\section{Summary}

This paper studies stationary and nonstationary distributions of money. For this purpose it relaxes the one-storage technology imposed in the early versions of the random-matching model of money by considering a model where agents can accumulate money up to the bound $n \in \mathbb{N}^{+}$. The following results emerge from the model. First, the quantity of money $M=\frac{n}{2}$ and the uniform distribution $m_{i}=\frac{1}{n+1}, i=0, \ldots, n$, maximize welfare. This result generalizes the welfare result of Kiyotaki and Wright $(1991,1993)$, where, in the absence of barter trades, the optimum amount of money is $\frac{1}{2}$. Second, if $C \leq \bar{C}$, any initial distribution of the optimum amount of money converges asymptotically to the uniform distribution of money holdings. This result, although derived under the assumption of given prices, supports Friedman's conjecture about the long-run irrelevance of the distribution of money. Although Friedman does not discuss this issue in detail, his conjecture is that a nonstationary distribution "will introduce initial distribution effects. During the transition, some men will have net gains in consumption, others net losses in consumption. But the ultimate position will be the same, not only for the aggregate, but for each individual separately" (1969, p. 6). 


\section{Appendix}

Proof of Lemma 1 In a steady state, the measure of agents who leave state $i$ equals the measure of agents that enter this state. All steady state conditions are summarized below:

$$
\begin{array}{rlrl}
n: & p_{b} m_{n} & =p_{s} m_{n-1} \\
n-1: & & \left(p_{b}+p_{s}\right) m_{n-1} & =p_{b} m_{n}+p_{s} m_{n-2} \\
& \vdots \\
1: & & \left(p_{b}+p_{s}\right) m_{1} & =p_{b} m_{2}+p_{s} m_{0} \\
0: & p_{s} m_{0} & =p_{b} m_{1}
\end{array}
$$

These conditions imply

$$
\begin{aligned}
p_{b} m_{i} & =p_{s} m_{i-1}, i=1, . ., n, \\
m_{i} & =\frac{m_{i-1}^{2}}{m_{i-2}}, i=2, . ., n
\end{aligned}
$$

Solving (15) recursively yields (2). Combine (2) with $\sum_{i=0}^{n} m_{i}=1$ to get (3). Then, divide $m_{i}=m_{0}^{\left(\frac{n-i}{n}\right)} m_{n}^{\left(\frac{i}{n}\right)}$ by $m_{i+1}=m_{0}^{\left(\frac{n-i-1}{n}\right)} m_{n}^{\left(\frac{i+1}{n}\right)}$ to get $\frac{m_{0}}{m_{n}}=\left(\frac{m_{i}}{m_{i+1}}\right)^{n}$. This and (14) yield (4).

I next show uniqueness of the stationary probability measure $m$ for any $n$ and money supply $M \in(0, n)$. The first thing to note is that (3) implies $\frac{\partial m_{n}}{\partial m_{0}}<0$. Thus, for any $n$ and $m_{0}$ there is a unique $m$ that satisfies (2) and (3). Next, note that (4) implies that $m_{0}$ is monotonically decreasing in $M$ (to see this note that the right-hand side of (4) is strictly decreasing in $m_{0}$; thus, an increase in $m_{0}$ reduces the ratios $\left.\frac{m_{i}}{m_{i-1}}, i=1, \ldots, n\right)$. Accordingly, for any $n$ and $M \in[0, n]$ there is a unique probability measure $m$ satisfying (2) and (3).

Proof of Lemma 2 The proof involves two steps. First, the critical value $\tilde{C}$ is derived. Second, existence and uniqueness are shown for $C \leq \tilde{C}$. 
First step. When all agents with money holdings $i<n$ produce and sell for money, the system of value functions (1) is reduced to

$$
\begin{aligned}
r V^{0} & =p_{s}\left(V^{1}-V^{0}-C\right) \\
r V^{i} & =p_{b}\left(U+V^{i-1}-V^{i}\right)+p_{s}\left(V^{i+1}-V^{i}-C\right), i=0, \ldots, n-1 \\
r V^{n} & =p_{b}\left(U+V^{n-1}-V^{n}\right) .
\end{aligned}
$$

(16) defines a second-order linear nonhomogeneous difference equation with constant coefficients and constant term and two initial conditions. The second equation is the difference equation, and the first and third equations are the initial conditions. The solution is

$$
V^{i}=\phi_{1} \lambda_{1}^{i}+\phi_{2} \lambda_{2}^{i}+\mu
$$

where

$$
\begin{gathered}
\lambda_{1}, \lambda_{2}=\frac{p_{b}+p_{s}+r \mp \sqrt{\left(p_{b}+p_{s}+r\right)^{2}-4 p_{s} p_{b}}}{2 p_{s}} \\
\phi_{1}=\frac{\left(1-\lambda_{2}\right)\left(p_{b} \lambda_{2}^{n} U-p_{s} \lambda_{1} C\right)}{r\left(\lambda_{2}^{n+1}-\lambda_{1}^{n+1}\right)}, \phi_{2}=\frac{\left(1-\lambda_{1}\right)\left(p_{s} \lambda_{2} C-p_{b} \lambda_{1}^{n} U\right)}{r\left(\lambda_{2}^{n+1}-\lambda_{1}^{n+1}\right)} \\
\mu=\frac{p_{b} U-p_{s} C}{r}
\end{gathered}
$$

One can show that the roots satisfy $0<\lambda_{1}<1$ and $\lambda_{2}>1$.

Note that $V^{i}$ is concave, i.e., $V^{i}-V^{i-1}-\left(V^{i+1}-V^{i}\right) \geq 0, i=1, . ., n-1$. Thus, it is sufficient to show that agents with money holdings $n-1$ produce for money, i.e.,

$$
V^{n}-V^{n-1} \geq C
$$

$\tilde{C}$ is the value of $C$ such that (21) holds with equality, which implies that if $C \leq \tilde{C}$ then $I \geq n$. Use (17), (18), (19), and (20) to get $\tilde{C}=\frac{\lambda_{1}^{n} \lambda_{2}^{n}\left(\lambda_{2}-\lambda_{1}\right) U}{\lambda_{1}^{n}\left(1-\lambda_{1}\right)-\lambda_{2}^{n}\left(1-\lambda_{2}\right)}$.

Second step. Consider, first, condition i) of Definition 1, consider any $n$ and $M \in$ $(0, n)$, and assume that $m$ is the unique stationary distribution characterized in Lemma 1. Then, for $C \leq \tilde{C}$, agents are willing to accumulate money up to the bound $n$. Consider, 
next, condition ii). When agents are willing to sell for money up to the bound $n$, by Lemma $1 \mathrm{~m}$ is the unique stationary probability measure we are looking for. Consider, finally, condition iii). Because $p_{s}>0$ and $p_{b}>0$, we have $V>0$. 


\section{References}

Berentsen, A., "Money Inventories in Search Equilibrium," Journal of Money, Credit, and Banking 32 (2000), 168-178.

Berentsen, A., M. Molico and R. Wright, "Indivisibilities, Lotteries, and Monetary Exchange," Journal of Economic Theory (forthcoming).

Berentsen, A. and G. Rocheteau, "On the Efficiency of Monetary Exchange: How Divisibility of Money Matters,” Journal of Monetary Economics (2001, forthcoming).

Berentsen, A. and G. Rocheteau, "Money and Terms of Trade," International Economic Review (forthcoming).

Camera, G. and D. Corbae, "Money and Price Dispersion," International Economic Review 40 (1999), 985-1008.

Friedman, M., The Optimum Quantity of Money and other Essays (Chicago: Aldine, 1969). Green, E. J., and R. Zhou, "A Rudimentary Random-Matching Model with Divisible Money and Prices," Journal of Economic Theory 81 (1998a), 252-71.

Green, E. J. and R. Zhou, "Monetary Equilibrium from an Initial State: the Case Without Discounting," mimeo, Federal Reserve Bank of Minneapolis and University of Pennsylvania, 1998b.

Lagos, R. and R. Wright, "A Unified Framework for Monetary Theory and Policy Analysis," mimeo (2001).

Kiyotaki, N. and R. Wright, "A Contribution to the Pure Theory of Money," Journal of Economic Theory 53 (1991), 215-34.

Kiyotaki, N. and R. Wright, "A Search Theoretic Approach to Monetary Economics," American Economic Review 83 (1993), 63-77.

Molico, M., "The Distribution of Money and Prices in Search Equilibrium," mimeo, Pennsylvania State University, 1998.

Rocheteau, G., "La Quantité Optimale de Monnaie dans un Modèle avec Appariements 
Aléatoires," Les Annales d'Economie et Statistique 58 (2000), 101-142.

Shi, S., "Money and Prices: A Model of Search and Bargaining," Journal of Economic Theory 67 (1995), 467-496.

Shi, S., "A Divisible Search Model of Fiat Money," Econometrica 65 (1997), 75-102.

Shi, S., "Search, Inflation and Capital Accumulation," Journal of Monetary Economics 44 (1999), 81-103.

Stokey, N.L. and R.E. Lucas, Recursive Methods in Economic Dynamics (Harvard University Press, Cambridge, Massachusetts, and London, England, 1989).

Taber, A. and N. Wallace, "A Matching Model with Bounded Holdings of Indivisible Money," International Economic Review 40 (1999), 961-984.

Trejos, A. and R. Wright, "Search, Bargaining, Money, and Prices," Journal of Political Economy 103 (1995), 118-141.

Zhou, R., "Individual and Aggregate Real Money Balances in a Random-Matching Model," International Economic Review 40 (1999), 1009-1038. 